\title{
Dynamic viscosity, centrifugation test and kinetic investigation in emulsions with pumpkin oil
}

\author{
Vanya Gandova ${ }^{1, *}$, Ivalina Petrova $^{2}$, Stanislava Tasheva ${ }^{3}$ and Albena Stoyanova ${ }^{4}$ \\ ${ }^{1}$ Department of Analytical and Physical chemistry, University of Food technologies, 4002 Plovdiv, Bulgaria \\ ${ }^{2}$ Department of Mathematics, Physics and Chemistry, Technical University Sofia, branch Plovdiv, 4002 Plovdiv, Bulgaria \\ ${ }^{3}$ Department of Heat Engineering, University of Food technologies, 4002 Plovdiv, Bulgaria \\ ${ }^{4}$ Department of Technology of Tobacco, Sugar, Vegetable and Essential Oils, University of Food Technologies, 4002 Plovdiv, \\ Bulgaria
}

\begin{abstract}
The emulsion stability of different pumpkin $\mathrm{O} / \mathrm{W}$ emulsions was investigated. Preparation of emulsions were performed with oil phase between 10 and 40 percent, water and soybean protein isolate. To determine emulsion properties different methods were used. Density, viscosity, centrifugation tests and spectroscopic measurements were performed for their investigations. The emulsions were stored for two weeks to determine their kinetic. Influence of the oil phase was presented and connected with the stability of them.
\end{abstract}

\section{Introduction}

Thermodynamics of emulsions is very unstable. The preparation and stabilization of emulsions is connected to protect them using different methods [1, 2]. Their formation exhibits action between surfactant or protein and the other components of the oil compound. The oil phase of an emulsion is correlated with the viscosity [3]. When added the water phase in them observed some instability in the system. After observed application in emulsions it is insisted for suitable physical and mechanical properties [4]. The formation of water-in-oilin-water emulsions in the hydrophilic or hydrophobic part of them are investigated. The influence of hydrophobic surfactant $(\mathrm{Span} \circledast 80)$ or $\mathrm{NaCl}$ and the particle size of the prime emulsions was presented. The smaller particle size about $0.51 \mu \mathrm{m}$ was observed [5-7].

Pumpkin seed oil is a source of biologically active compounds and fatty acids. For preparation of water-inoil emulsions droplet size and surface properties were used to obtain optimal conditions. Emulsion droplets exhibit a diameter in a range between 400 and $850 \mathrm{~nm}$ [8]. Surface active compounds such as free fatty acids, phospholipids, and chlorophyll are the main in pumpkin seed oil and they adsorbed on the membrane. Wetting of membranes connected with the adsorption of these components. The oil phase leads to the formation of the drops with same size [9].

Influence of chemical composition and $\mathrm{pH}$ values in emulsions prepared with pumpkin seed oil and raw and cooked chicken meat lead to producing health meat products with low lipids [10].

Natural emulsions for oral administration, incorporating pumpkin seed oil and hemp seed oil with lecithin as emulsifying agent were observed. Using the analysis with approaches to a drop model two types of emulsions $\mathrm{O} / \mathrm{W}$ and $\mathrm{W} / \mathrm{O}$ were prepared and characterized in sensory parameters, conductivity properties and superficial analysis. Surface tension of lecithin was analyzed and provided information about proper stability. The surface tension values are found around $25 \mathrm{mN} \cdot \mathrm{m}^{-1}$. The contact angle was measured too and values are between $31.87 \pm 0.51$ and $44.01 \pm 5.48$. It is found that wettability correlates with the internal structure [11].

Stable O/W emulsions were obtained by plant seed oil. The cream of emulsions provides different effects in the emulsion systems. The synergistic effect of the pumpkin seed oil blended with vitamin $\mathrm{E}$ used to cream formulation. Different analysis for physical properties, antioxidant activities and storage stability were provided [12].

In the literature do not find data for the influence of the pumpkin seed oil as oil phase on the stability of food emulsions.

This study aimed to investigate the influence of the oil phase in emulsions and presented characteristics such as viscosity, centrifugation test and storage period on them.

\section{Materials and Methods}

All investigations were provided with twelve emulsions prepared with pumpkin oil. For stabilizer was used soybean protein isolate (SI). Different percent water was used to prepare emulsions. Mixture between water, oil and protein was homogenized for $2 \mathrm{~min}$ at room temperature $\left(25^{\circ} \mathrm{C}\right)[13]$.

Viscosity determination appeared as an important characteristic of liquids. Dynamic viscosity was determined using vertical falling ball viscometer. Speed of ball was determined with spindle number 5 . The

\footnotetext{
*Corresponding author: gandova $71 @$ abv.bg
} 
dynamic viscosity was calculated including experimental density and Stokes law [14].

$$
\eta=\frac{2}{9} \frac{\left(\rho_{e m}-\rho_{b}\right) g r^{2}}{v_{0}}
$$

where: $\mathrm{g}$ - gravity, $\mathrm{m} . \mathrm{s}^{-2} ; \mathrm{r}$ - radius of the spheres, $\mathrm{m}$; $\rho_{\mathrm{em}}-$ emulsion density, $\mathrm{kg} . \mathrm{m}^{-3} ; \rho_{\mathrm{b}}-$ density of the spheres, kg. $\mathrm{m}^{-3} ; \eta$ - viscosity, Pa.s; $v_{0}-$ speed determined by the unit time.

Density was measured by a pycnometer. Generally, exhibit values around 0.88 to 0.83 . Stability of emulsions connected with the physical structure of the emulsion system.

Centrifugation test was performed for the twelve emulsions. Centrifugation measurements determined mechanical properties of them. Prepared emulsions are heating at constant temperature $45^{\circ} \mathrm{C}$ and for a determined time. They are centrifuged for $5 \mathrm{~min}$ at speed $6000 \mathrm{rpm}$.

After a centrifugation test the physical stability was determined using the eq. (2) [15].

$$
E P E=\frac{V U P}{T E V}
$$

where: EPE - emulsion phase; VUP -volume of upper phase, $\mathrm{cm}^{3}$; TEV - total emulsion volume, $\mathrm{cm}^{3}$.

Spectrophotometer measurements was provided to determined emulsion stability during the storage period by turbidity measurements. For the provide experiment wavelength was determined at $450 \mathrm{~nm}$. A Camspec UV/VIS spectrophotometer M107 was used [14]. The change in the emulsion turbidity was observed from 1-14 days. First measurement was provided immediately after their preparation.

The equation of linear regression was used and presented as eq. 3 :

$$
y=67.63+1.5 x
$$

where: $\mathrm{x}$ - emulsion phase stability; $\mathrm{y}$ - experimental viscosity.

All measurements were performed in triplicate. The standard deviation (SD) after provided experiments was determined using Origin Pro 7 software.

\section{Results and Discussion}

Emulsions with pumpkin oil exhibit good application in foods. Physical and mechanical characteristics of them determined their stability. In preparation a large amount of energy is required. Application by Stokes law was used with the experimental density [16]. Using density for emulsion stability dynamic viscosity was calculated. The obtained results are presented in Table 1.

According literature data viscosity of pure Pumpkin oil is determined with the following value $\eta=70 \pm 0.47$ $10^{3} \mathrm{~Pa} . \mathrm{s}$ [15]. In the Table 1 viscosity of emulsions exhibit values comparative with a pure pumpkin oil as they rise slightly. This small differences are connected with protein addition necessary to improve emulsion stability.
Connection between density and viscosity according eq. 1 is observed. With increasing density, the viscosity increases too.

The viscosity of emulsions is strongly correlated at different oil percentages. The figure shows that with increase in the oil phase increase and the viscosity. In this case emulsion stability improves at high oil content and high viscosity $[3,6]$.

Table 1. Experimental density and viscosity on emulsions with pumpkin seed oil.

\begin{tabular}{|c|c|c|c|}
\hline Emulsions & $\boldsymbol{\rho}$ & $\boldsymbol{\eta}$ Pa.s & $\boldsymbol{\eta} \mathbf{1 0}^{\mathbf{3}}$ Pa.s \\
\hline 1 & $0.884 \pm 0.02$ & $0.094 \pm 0.21$ & $93.676 \pm 0.13$ \\
\hline 2 & $0.875 \pm 0.02$ & $0.095 \pm 0.20$ & $94.912 \pm 0.11$ \\
\hline 3 & $0.869 \pm 0.01$ & $0.104 \pm 0.21$ & $104.494 \pm 0.14$ \\
\hline 4 & $0.854 \pm 0.01$ & $0.113 \pm 0.22$ & $112.871 \pm 0.10$ \\
\hline 5 & $0.881 \pm 0.02$ & $0.105 \pm 0.18$ & $105.322 \pm 0.12$ \\
\hline 6 & $0.870 \pm 0.01$ & $0.109 \pm 0.15$ & $108.855 \pm 0.18$ \\
\hline 7 & $0.868 \pm 0.02$ & $0.128 \pm 0.16$ & $128.090 \pm 0.21$ \\
\hline 8 & $0.855 \pm 0.02$ & $0.131 \pm 0.20$ & $131.212 \pm 0.17$ \\
\hline 9 & $0.873 \pm 0.02$ & $0.114 \pm 0.18$ & $113.765 \pm 0.19$ \\
\hline 10 & $0.858 \pm 0.01$ & $0.126 \pm 0.17$ & $125.813 \pm 0.15$ \\
\hline 11 & $0.844 \pm 0.01$ & $0.128 \pm 0.21$ & $128.022 \pm 0.12$ \\
\hline 12 & $0.831 \pm 0.01$ & $0.135 \pm 0.21$ & $134.711 \pm 0.11$ \\
\hline
\end{tabular}

$\rho$ - density; $\eta$ - viscosity

The dependence between oil phase in emulsions and viscosity was presented on Fig. 1.

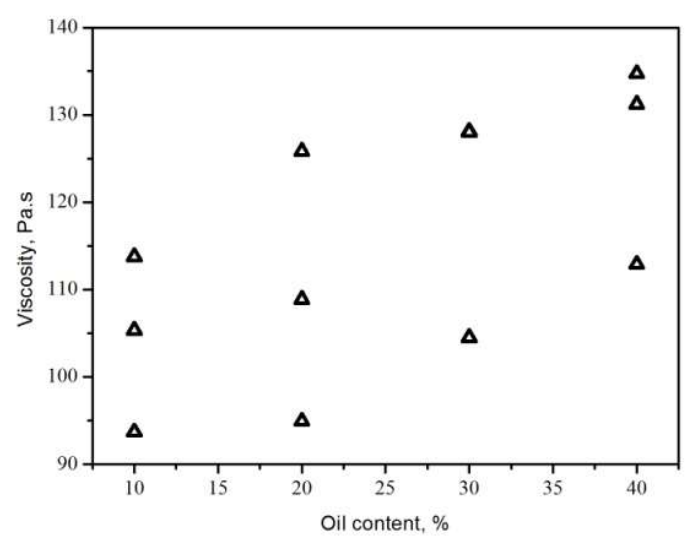

Fig. 1. Correlation between starting oil and emulsion viscosities.

The centrifugation test in emulsion systems used to determine stability from the separation level [4]. The lower separation does the high stability of emulsion systems [5]. As a starting point this test played a role in the physical characteristics of emulsions [5-7].

Centrifugation test for all emulsions was provided for their investigation and after experiment three phases were observed. Table 2 presented a description of obtained phases and weight of emulsion samples. As a result, after centrifugation two main phases $\mathrm{O} / \mathrm{W}$ and $\mathrm{W} / \mathrm{O}$ first appeared. In a third part oil phase was observed. According to the authors [15], the quantity of protein deposition of all emulsions was seen. Graphical view between phases after centrifugation test is presented on Fig. 2. It is seen that with the increase of oil phase $\mathrm{O} / \mathrm{W}$ emulsions appeared as a high part. 
Table 2. Observed phases in the emulsions after the centrifugation test.

\begin{tabular}{|c|c|c|c|c|}
\hline \multirow{2}{*}{ Emulsions } & \multicolumn{4}{|c|}{ Phases, $\mathbf{~ c m}^{\mathbf{3}}$} \\
\cline { 2 - 5 } & $\mathbf{1}$ & $\mathbf{2}$ & $\mathbf{3}$ & $\mathbf{4}$ \\
\hline 1 & $3.5 \pm 0.01$ & $2.0 \pm 0.03$ & $1.5 \pm 0.05$ & 15 \\
\hline 2 & $2.3 \pm 0.01$ & $2.5 \pm 0.02$ & $1.2 \pm 0.01$ & 15 \\
\hline 3 & $2.2 \pm 0.02$ & $2.4 \pm 0.04$ & $1.9 \pm 0.07$ & 15 \\
\hline 4 & $2.2 \pm 0.01$ & $2.3 \pm 0.04$ & $2.2 \pm 0.01$ & 18 \\
\hline 5 & $4.0 \pm 0.02$ & $1.6 \pm 0.01$ & $1.8 \pm 0.04$ & 18 \\
\hline 6 & $3.4 \pm 0.01$ & $1.8 \pm 0.01$ & $1.9 \pm 0.01$ & 13 \\
\hline 7 & $2.8 \pm 0.01$ & $2.0 \pm 0.01$ & $2.5 \pm 0.02$ & 15 \\
\hline 8 & $3.0 \pm 0.01$ & $1.4 \pm 0.03$ & $3.9 \pm 0.02$ & 20 \\
\hline 9 & $2.5 \pm 0.01$ & $1.8 \pm 0.01$ & $1.5 \pm 0.03$ & 11 \\
\hline 10 & $2.8 \pm 0.01$ & $1.9 \pm 0.04$ & $2.5 \pm 0.06$ & 17 \\
\hline 11 & $3.5 \pm 0.02$ & $1.9 \pm 0.03$ & $3.4 \pm 0.05$ & 20 \\
\hline 12 & $2.9 \pm 0.02$ & $1.9 \pm 0.01$ & $3.9 \pm 0.05$ & 20 \\
\hline
\end{tabular}

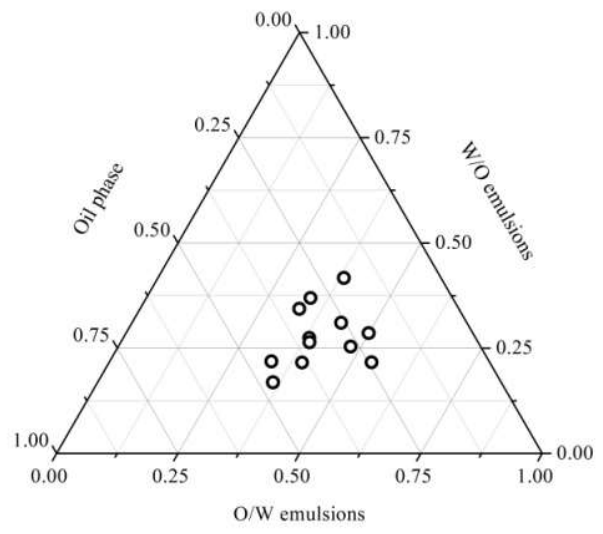

Fig. 2. Graphical presentation between different phases after centrifugation test.

After centrifugation test the physical stability of emulsions was predicted using experimental results described in Table 2. By eq. 2 the emulsion phase stability was calculated and results are between 0.21 to 0.44 . Emulsions prepared with high oil percent exhibit a large phase stability. After all, obtained results connected with emulsion stability can be concluded that emulsions with 30 and 40 oil percent are more stable. Different dependencies were observed. Some of them for example dependence between emulsion phase stability and viscosity of emulsions was presented on Fig. 3.

After regression the high coefficient of correlation is obtained $\mathrm{R}^{2}=0.9202$. This indicates the good correlation between emulsion phase stability and viscosity.

After that another measurement proceeds during fourteen days. The obtained results gave values between 6.4 after preparation to 3.7 in fourteen days. The dependence for turbidity per day can be seen in Fig. 4. In the figure is observed high turbidity in first to third day, about 7.5. After following days turbidity decreased and after that to fourteen day presented approximately constant value about 3.9 to 3.7 at the end.

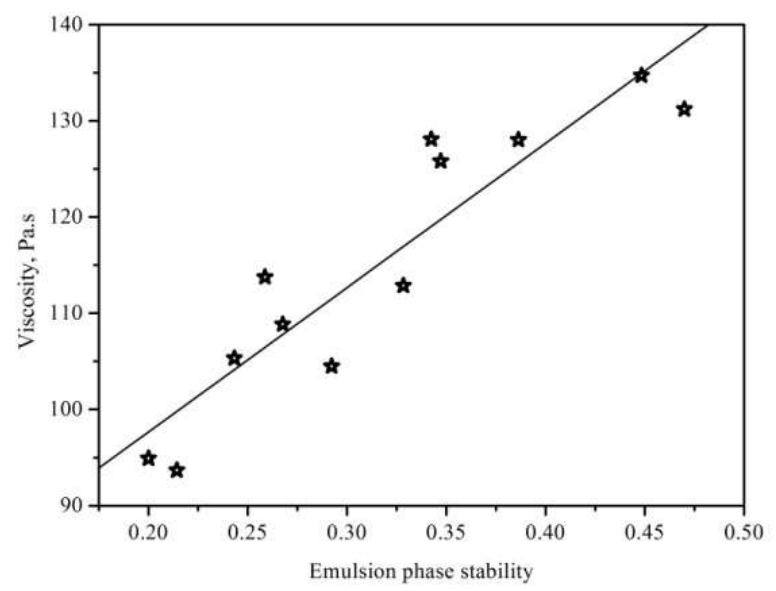

Fig. 3. Dependence between emulsion stability and viscosity in emulsions.

Turbidity measurements connected with emulsion stability too. According to observed results some stable emulsions will appear on the third day. So long storage periods are not recommended for investigation of emulsion stability [5].

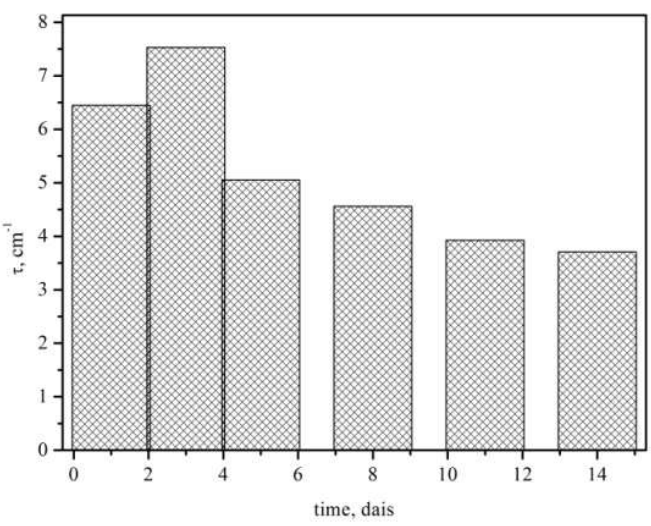

Fig. 4. Turbidity in days on investigation emulsions.

\section{Conclusion}

Investigated stability on twelve emulsions were presented using physical and physicochemical methods. Emulsions prepared with mixing of oil and water phases with protein addition. Influents of oil phase show that the emulsions with high oil percent 30 and 40 show good stability. Using the multiple linear regression model, the correlation between emulsion stability and viscosity was obtained. A high coefficient of correlation was observed $\mathrm{R}^{2}=0.9202$ after obtaining the equation. After spectrophotometer measurements, turbidity of twelve emulsions were obtained. Turbidity of emulsions connected with their stability too. Some high turbidity is observed on the third day after storage period. This result confirms that the storage period for prepared emulsions does not apply for a long time. 
Acknowledgements: The manuscript was supported by Project 04/20-H, University of Food Technologies, Plovdiv, Bulgaria and authors gratefully acknowledge.

\section{References}

1. C. Jadhav, V. Vaishali, S. Payghan, Nanostruct. Chem. 5, 107 (2015)

2. B. França, V. D’Amato, T. Esteves, A. Uller, F. Pessoa, Chem. Engin. Trans. 24, 745 (2011)

3. M. Fingas, B. Fieldhouse, M. Bobra, E. Tennyson, Proc. of the workshop on emulsions. The physics and chemistry of emulsions (Marine Spill Response Corporation, Washington, 1993)

4. C. M. Chen, C. H. Lu, C. H. Chang, Y. M. Yang, J. R Maa, Physicochem. Engin. Asp. 170, 173 (2000)

5. M. Artiga-Artigas, A. Molet-Rodríguez, L. SalviaTrujillo, O. Martín-Belloso, Food Bioproc. Technol. 12, 422 (2019)

6. O. Rybak, Ukrainian Food J., 5, 499 (2016).

7. V. Ivanov, O. Shevchenko, A. Marynin, V. Stabnikov, O. Gubenia, O. Stabnikova, A. Shevchenko, O. Gavva, A. Saliuk, Ukrainian Food J., 10, 7 (2021)

8. B. Nikolovski, J. Ilić, M. Sovilj, M. Nikolić, J. Milanović, Acta Period. Techn. 42, 175 (2011)

9. M. Dragosavac, M. Sovilj, S. Kosvintsev, R. Holdicnh, G. Vladisavljevic, J. Membr. Sci. 322, 178 (2008)

10. M. Serdaroğlu, B. Nacak, M. Karabıyıkoğlu, M. Tepe, I. Baykara, Y. Kökmen, IOP Conf. Series: Earth Environ. Sci. 85, 012045 (2017)

11. M. C. Anicescu, C. E. Dinu-Pirvu, M. V. Ghca,V, Anuta, R. M. Prisada, M. T. Talianu, L. M. Popa, Proc. $8^{\text {th }}$ Int. Con. Adv. Mater. Syst. (Notational Research \& Development Institute for Textiles and leather (INCDTP ICPI), Bucharest, 2020)

12. T. S. Ong, C. C. Chu, C. P. Tan, K. L. Nyam, J. Oleo Sci. 69, 297 (2020)

13. V. Gandova, S. Tasheva, I. Petrova, K. Marinova, M. Stoyanova, A. Stoyanova, Oxid. Commun. 44, 13 (2021).

14. V. Gandova, S. Tasheva, K. Dobreva, V. ProdanovaStefanova, I. Dincheva, M. Dimitrova, A. Stoyanova, Oxid. Comm. 43, 220 (2020)

15. K. Laidler, J. Meiser, Physical chemistry (BenjaminCummings Publishing Company, San Francisco, 1982)

16. J. Tsaknis, S. Lalas, E. Lazos, Gras. Aceit. 48, 267 (1997)

17. Y. Navarro-Pérez, E. Cedeño-Linares, O. NormanMontenegro, V. Ruz-Sanjuan, Y. Mondeja-Rivera, A. Hernández-Monzón, M. González-Bedia, J. Pharm. Pharmac. Res. 9, 98 (2021) 